\title{
A comparative study on the predictive ability of the decision tree, support vector machine and neuro-fuzzy models in landslide susceptibility mapping using GIS.
}

\begin{abstract}
he purpose of the present study is to compare the prediction performances of three different approaches such as decision tree (DT), support vector machine (SVM) and adaptive neurofuzzy inference system (ANFIS) for landslide susceptibility mapping at Penang Hill area, Malaysia. The necessary input parameters for the landslide susceptibility assessments were obtained from various sources. At first, landslide locations were identified by aerial photographs and field surveys and a total of 113 landslide locations were constructed. The study area contains 340,608 pixels while total 8403 pixels include landslides. The landslide inventory was randomly partitioned into two subsets: (1) part 1 that contains $50 \%$ (4000 landslide grid cells) was used in the training phase of the models; (2) part 2 is a validation dataset 50\% (4000 landslide grid cells) for validation of three models and to confirm its accuracy. The digitally processed images of input parameters were combined in GIS. Finally, landslide susceptibility maps were produced, and the performances were assessed and discussed. Total fifteen landslide susceptibility maps were produced using DT, SVM and ANFIS based models, and the resultant maps were validated using the landslide locations. Prediction performances of these maps were checked by receiver operating characteristics (ROC) by using both success rate curve and prediction rate curve. The validation results showed that, area under the ROC curve for the fifteen models produced using DT, SVM and ANFIS varied from 0.8204 to 0.9421 for success rate curve and 0.7580 to 0.8307 for prediction rate curves, respectively. Moreover, the prediction curves revealed that model 5 of DT has slightly higher prediction performance (83.07), whereas the success rate showed that model 5 of ANFIS has better prediction (94.21) capability among all models. The results of this study showed that landslide susceptibility mapping in the Penang Hill area using the three approaches (e.g., DT, SVM and ANFIS) is viable. As far as the performance of the models are concerned, the results appeared to be quite satisfactory, i.e., the zones determined on the map being zones of relative susceptibility.
\end{abstract}

Keyword: Landslides; Decision tree (DT); Support vector machine (SVM); Adaptive neurofuzzy inference system (ANFIS); Malaysia; GIS; Remote sensing 